Research Article

\title{
Current Status of Ceramic Industry and VR Technology Used in Ceramic Display and Dissemination
}

\author{
Xue Li ${ }^{1}{ }^{1}$ and Xiaobing $\mathrm{Hu}\left(\mathbb{D D}^{2}\right.$ \\ ${ }^{1}$ School of Art and Design, Jingdezhen Ceramic University, Jingdezhen 333000, Jiangxi, China \\ ${ }^{2}$ Fine Art School, Anqing Normal University, Anqing 246001, Anhui, China \\ Correspondence should be addressed to Xiaobing Hu; 315698886@qq.com
}

Received 16 August 2021; Accepted 9 October 2021; Published 26 October 2021

Academic Editor: Punit Gupta

Copyright (c) 2021 Xue Li and Xiaobing Hu. This is an open access article distributed under the Creative Commons Attribution License, which permits unrestricted use, distribution, and reproduction in any medium, provided the original work is properly cited.

\begin{abstract}
With the deepening of reform and opening up, the development of China's ceramic industry has been rapidly improved, leading the world, and various ceramic varieties have also been greatly developed. However, as the growth rate of the global economy has gradually slowed down and structural imbalances have become more obvious, China's economy has gradually entered a new development trend. In the context of supply-side structural reforms, the severe macroenvironment and policy pressure to eliminate backward production capacity have further promoted the development of China's ceramic industry to face greater challenges. In the context of the rapid development of various high-tech technologies such as "Internet +" and intelligent manufacturing, this paper discusses the use of VR technology in the design of ceramics from the principles and characteristics of ceramic design and, according to the characteristics of virtual design of ceramics, demonstrates the feasibility of its shape, decoration, color matching, and so on. The ceramics are classified according to their use functions, and the characteristics of different types of virtual display of ceramics and their suitable virtual display methods are discussed. Finally, this paper combines panoramic image display technology and graphic VR display technology to create the best virtual display method suitable for different types of ceramic products, implements the interactive design in virtual software, and then performs virtual display.
\end{abstract}

\section{Introduction}

Over the past 30 years of reform and opening up, China's ceramic industry has developed rapidly. China has taken the forefront of ceramic development, becoming the center of ceramic manufacturing and the main producer of ceramics, with the first annual output and export. Chinese daily ceramics are about $70 \%$ of the world, $65 \%$ and about $50 \%$ of sanitary ceramics, and $64 \%$ of construction ceramics. Due to the obvious labor cost and resource advantages, the competitiveness of China's ceramic industry is also being rapidly improving, and its position in the world ceramic market is being rapidly improving.

In recent years, China's ceramic industry has grown rapidly at an average annual rate of more than $20 \%$, is at the middle development level in all industries of the national economy, and far exceeds the growth rate of GDP. The ceramic industry has developed into one of the important industries to promote the sustainable, steady, and healthy development of China's national economy.

Despite the continuous improvement of the technology and development of China's ceramic industry, the domestic and foreign markets have grown rapidly and have achieved good results. However, as the global economic growth rate gradually slows down and the structural imbalance becomes more obvious, China's economy has gradually entered a new development trend. In the context of supply-side structural reform, the severe macroenvironment and the policy pressure of eliminating backward production capacity further promote the development of China's ceramic industry that is facing greater challenges. However, with the continuous improvement of Chinese residents consumption level, "Internet +," and intelligent manufacturing rapid development of various high-tech technology, as well as the 
rapid implementation of the development of ceramic industry special planning, China's ceramic industry ushered in a good development opportunity, supporting the development of China's ceramic industry in the future in a good and rapid direction.

This paper mainly analyzes the development status of China's ceramic industry in recent years in a narrative manner and combines the advantages of VR technology in the promotion and display of the ceramic industry to do research. Based on the application of VR technology in ceramics, some scholars have made some comments from different perspectives. These studies can be roughly divided into three categories. One is the analysis of the local kiln opening display from a case study [1], which focuses on the use of VR technology to display ancient cultural relics. The point of view is that the display of ancient cultural relics in the real scene is likely to cause damage to the artifacts, and not only can the use of VR technology satisfy people's appreciation of ancient cultural relics, especially in the postepidemic era, but also people can display them in VR without leaving their homes. Feel the charm of ceramics in the virtual space. One is the use of VR technology in the design of ceramic products [2], mainly discussing how to apply cutting-edge VR technology to improve users' interactive virtual experience of products on e-commerce platforms. Solve the contradiction between mass production and individual differences in consumption; at the same time, it discusses how to use it to realize the reproduction, repair, and data storage of ceramic products and how to realize the construction of a virtual inspection and evaluation system for ceramic products; third class is the application of VR technology in modern ceramic display design [3]. The point of view is that the ceramic exhibition hall is a platform to showcase the ceramic culture and disseminate ceramic art to the audience. As the ceramic capital of millennia, Jingdezhen ceramic art has been promoted to a large extent Thanks to the development of exhibition art, with the development of technology, virtual reality technology is also promoting the development of ceramic exhibition halls. The current basic characteristics of immersion, interactivity, and sharing of virtual reality technology are designed for ceramic exhibition halls. Work brings new inspiration and vitality. The application of VR technology to the design of ceramic exhibition halls is bound to be the development trend of ceramic exhibition hall exhibition design in the future. The above-mentioned research results are to explain the application of VR technology in ceramics display and design in a small range, and the application methods and technical points of VR technology had not been involved in the analysis of China's ceramic industry; this will be the focus of this paper.

\section{Ceramic Development Environment and Influence}

2.1. Impact of the International Economic Environment on China's Ceramic Industry. After the outbreak of the US financial subprime crisis in 2008, the global scope further expanded, triggering a global financial crisis, and the global economy is gradually entering a period of deep adjustment. In the coming extraordinary period, global economic development will generally recover. Under this background, there will still be greater uncertainty and instability. The global trade environment has also become worse, and it is unlikely to improve in the short term. Moreover, with the introduction of the TPP trade agreement headed by the United States, China, as the world's largest producer and exporter of ceramic products, has also been excluded from the agreement. With the arrival of US President Donald Trump, the TPP agreement was canceled during this period, which is also a very rare opportunity for the development of China's ceramic industry.

At the same time, we also need to see that the overall trend of international economic development presents new opportunities. World economic development focus gradually shifted from the established developed countries to the current emerging markets and developing countries or economies, industrialization and urbanization space, may form huge demand for production and life, and will have a great impact on the development demand and regional distribution of Chinese ceramic products. Developing countries and emerging economies are also playing an increasing position in China's ceramic industry exports.

However, the international market demand for daily ceramics has a trend of slowing down under the environmental impact of the global economy. However, the demand for high-grade and quality daily ceramics is increasing year by year. This situation has caused the international manufacturers of daily ceramics to focus on the high-grade and cultural and artistic characteristics of ceramics; those products with collection value and gift value, good quality, full function, and novel decoration are gradually favored. This situation is an opportunity for Chinese producers. Since ancient times, the ceramic industry has had a profound cultural heritage, the ceramic products in each producing area are distinctive, and the whole can produce complementary utility to seize the international market. China is a large import country of ceramics between the United States and the EU, USD 22,222 billion from China, accounting for $38.56 \%$ of the total ceramic imports, and $\$ 1.698$ billion from China in 2016, representing $43.67 \%$ of the total ceramic imports. From the total ceramic import output of the United States and the EU in 2016 (Figures 1 and 2), China has an obvious ceramic industry in these two regions.

\subsection{Influence of the Domestic Economic Environment on the} Ceramic Industry. As the Chinese market economy enters a structural slowdown, GDP slows from high development to medium development. China is also facing great downward pressure on the reverse of transformation and upgrading of economic structure. Under increasing economic downward pressure, decreasing marginal effect of monetary policy, increasing resource and environmental constraints, rising factor costs, and overcapacity industries, "three high" type pollution industries face "overcapacity," and the ceramic industry is also affected. Under the background of supplyside structural reform and development, the ceramic 


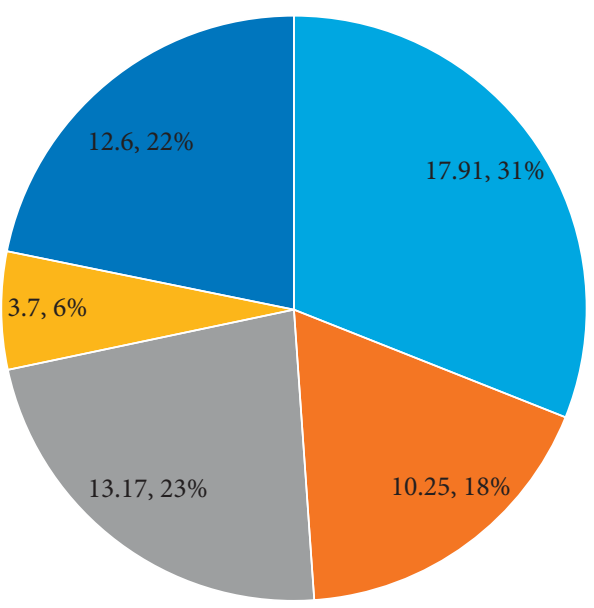

- Architectural ceramics

- Sanitary ceramics

- Daily-use ceramics

- Art ceramics

- Other types

Figure 1: Import of American ceramics in 2016.

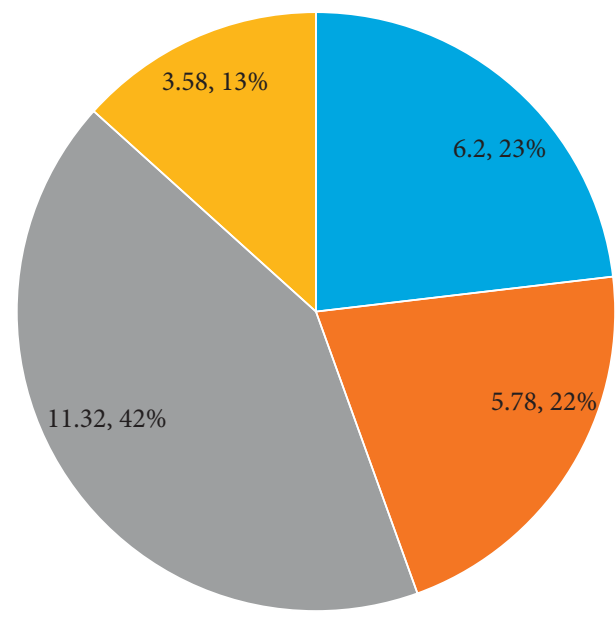

$$
\begin{aligned}
& \text { Architectural ceramics } \\
& \text { - Sanitary ceramics } \\
& \text { Daily-use ceramics } \\
& \text { - Art pottery }
\end{aligned}
$$

FIgURE 2: Import situation of EU ceramic products in 2016.

industry is bound to face the extremely severe market competition pressure and the huge constraints of the environment, and the transformation and upgrading will also become an inevitable choice for the ceramic industry in the future development process.

Over the years, the rapid growth of GDP has gradually become an important prerequisite for the stable growth of household income, covering the social security system of towns and villages, and specific consumption habits have also been initially formed. The current desire to upgrade consumption is extremely strong. In addition, in the context of accelerating the construction of new urbanization, new consumer demand has also created a new market for the transformation and upgrading of the ceramic industry. Based on the recovery of the real estate market, continue to promote the rapid recovery of the downstream building and health ceramics industry. During this period, new rigid demands and improved demands were also activated. The development of the ceramic industry is facing new development opportunities. The "Internet + ceramics" model has been continuously developed in the exploration of the modern market, which has promoted the rapid integration and development of the traditional ceramic industry and the emerging service industry and promoted the structural transformation and upgrading of the ceramic industry.

2.3. Development Environment and Technical Status in the Ceramic Industry. There are a large number of enterprises in the ceramic industry, and the average scale is relatively small, with weak research and innovation ability and low brand value. A series of problems have become very prominent at present, resulting in problems related to low-level repeated construction, unreasonable industrial layout, and the imbalance between supply and demand caused by the rapid growth of production capacity. In recent years, as the whole society has increased awareness of environmental governance, energy conservation, and emission reduction, the rapid increase of energy consumption and environmental protection costs restricts the development of the ceramic industry, because the problem has been "closed, stopped, merged, and transferred" production lines or those seeking pollution "haven" are gradually increasing. A large number of backbone enterprises with large scale, advanced technology, standardized management, strong brand awareness, and a strong sense of social responsibility in the industry have achieved good results in product quality, energy conservation, emission reduction, economic benefits, and many other aspects, and the industrial concentration degree has been gradually improved. However, as far as the overall operation and development level of ceramic enterprises, the number of enterprises that can participate in the whole industrial chain of the cooperation is not large. The marketoriented cooperation of the whole industrial chain still needs the further deepening and development of the ceramic industry. At the same time, China's ceramic industry has its typical disadvantages, mainly small and medium enterprises, difficult to quickly integrate into the global value chain. The competitive advantage is low factor cost and low tax incentives. Fusion positioning and homogenization of competition are very prominent; the low-level competitive advantage is prone to an antidumping investigation. In addition, small- and medium-sized ceramic exporters also have the risk of being replaced by lower-cost developing country enterprises, relying on low value-added products to support the industry's rapid growth model.

The technical environment of China's ceramic industry has been continuously improved, and the industrial technology research and development have basically entered a virtuous circle, but the intellectual property protection system still needs to be further improved. In the process of global competition, key technologies still need to be 
improved. In recent years, the construction of intellectual property protection system in China's ceramic industry has gradually tended to the overall benign development but is not ideal in intellectual property protection and law enforcement effect; the infringement still needs to be strengthened; the victim relief measures are still imperfect, resulting in the insufficient innovation motivation of small and medium-sized enterprises. "Promoting porcelain through science and technology" has gradually developed into a consensus of enterprises. Investment in research and development and technology promotion is constantly increasing, providing solid technical support for the transformation and upgrading of the industrial structure. To further improve the development quality and output efficiency of the building and healthy ceramic industry, prevent excessive growth, curb low-level repetitive construction, and promote the transformation and upgrading of the ceramic industry, relevant departments have also formulated or further revised a series of necessary development plans. Introduce the technical conditions for the development of smart ceramics and the basic conditions for its application. In addition to the various production technologies of the porcelain areas themselves, these technologies vigorously develop VR technology and AR technology, which are a more practical and scientific way to publicize and display Chinese ceramics in the world. Because VR technology does not need to work in the porcelain area and public display space, at home you can browse the representative ceramics in the virtual space with the Internet and computer, which effectively plays a role in promoting the publicity of Chinese ceramics.

\section{VR Technology and Application Methods}

3.1. VR Technology. VR technology is an advanced computer man-machine interface technology with basic features of immersion, interactivity, and conception, which integrates the science of human and information [4]. It comprehensively utilizes computer graphics, simulation technology, multimedia technology, artificial intelligence technology, computer network technology, parallel processing technology, and multisensor technology to simulate human vision, hearing, touch, and other sensory organ functions, so that people can be immersed in computer generation in the virtual realm and can interact with it in real time through natural means such as language and gestures, creating a humanized multidimensional information space. Users can not only experience the fidelity experienced in the objective object through the virtual reality system, so that people have a kind of "immersive" sense of reality, but also can break through the space, time, and other objective constraints and feel in the real world. Experience cannot be experienced personally [2].

Due to the main characteristics of VR technology such as immersion, interactivity, and conception, in the current society, especially in the postepidemic era, it has a very obvious advantage in the promotion and display of Chinese ceramics. In this process, companies use modern technological means of VR technology to achieve good interaction between users and products in the process of publicity and display in an effective way. Even under certain conditions, viewers can also use VR. Technology is involved in the design of ceramic products.

\section{2. $3 D$ Modeling of VR Technology Intervention Display.} The interactive characteristics of VR video refer to the audience's subjective consciousness and the right to operate things existing in space; on the other hand, virtual reality space accordingly gives natural and reasonable feedback and interaction behavior. The generation of VR video interactivity requires some supporting equipment such as using VR glasses, VR helmets, and data gloves so that the audience can feel the same in the real world through natural contact. The quality of the interactive hardware device affects the content delivered. It is because of the interaction function that determines that the effect of video delivery varies from person to person. The implementation of audience interaction in VR video also reflects the nature of the current interaction. This is also an improvement of Internet interaction that implements interactive behavior. Real-time feedback improves the value of the interaction behavior $[5,6]$.

SolidWorks is the mainstream solid modeling software based on parametric geometric features. SolidWorks uses geometric features as the design unit and uses geometric features to build part models. Geometric features are the basic units that make up a 3D model. In SolidWorks, geometric features are divided into sketch features and directly generated features according to different production methods. Before designing the model, it is necessary to decompose the complex model, establish a general function sequence, and clarify how to determine the sketch and reference level of each function [7].

Entity modeling software requires users to have certain $3 \mathrm{D}$ reverse thinking and can split a complex $3 \mathrm{D}$ model into groups of sketch features or directly generate feature combinations. At the same time, in the process of creating the basic features, how to choose the benchmark plane and how to choose the sketch plane are a test of the user's ability and experience. Polygon modeling software requires users to have a strong sense of space and space sense, and reasonable structure control ability, reasonable wiring ability, 3D model structure control ability, and 3D model grid distribution ability are also an index [8] to distinguish the level of polygon modeling ability.

\subsection{The Production Process of VR Technology Virtual Display} Design. The need for the virtual display of ceramic products is mainly based on the virtual reality display technology of 3D modeling. The display principle can be embodied by the following design and production steps:

(1) According to the purpose and content of the display, the designer uses 3D MAX, MAYA, and other types of three-dimensional modeling software to construct the digital model and make related optimizations. 
(2) Use the corresponding mechanism material materials to map or render the built ceramic product three-dimensional model, give the relevant scene lights in the virtual space, and then adjust the relevant parameters according to different space display requirements.

(3) Create animation effects after setting up the scene camera.

(4) After the first three parts are completed, save them in the corresponding format and then import them into the VR software for interactive design production, so that it has the function of the interactive operation. At the same time, multimedia information such as sound effects, text, and UI interface should be added.

(5) After the interactive production is completed, the output file can be used in practice according to the type of release.

The design process is shown in Figure 3.

3.4. Realization of VR Technology in Ceramic Display. The three-dimensional modeling design based on virtual reality display has its characteristics. Its rich display effect allows the audience to watch it. In addition to zooming in and out, it can also rotate at any angle, modify the selection of colors and patterns, and so on. The interactive performance is much better than the way of displaying panoramic video. Therefore, for the realization of VR technology in the display of ceramic products, we must first figure out how to encode and decode VR panoramic images. Among them, the process of encoding and decoding is essential to analyze and decode each frame for projection to realize panoramic video. We proceed as follows.

One is to decompose the panoramic video image into a single frame image. Since ceramic products are mostly threedimensional, it is most appropriate to analyze the ceramic display with the principle of panoramic projection of geometric spheres as a case. First, perform different projection formats according to different geometric models [9]; then, according to the actual situation, expand the geometry module [10] and again rearrange the geometry by different layout methods module; finally, the spherical image is converted into more conventional flat rectangular images, and these images are regarded as panoramic frames and correspond to the two-dimensional flat frames.

Second, the sequence of two-dimensional plane frames is encoded and compressed to obtain a data stream for video storage or transmission.

After the above two processes, the panoramic video encoding is completed. For decoding, it is to reverse the sequence of the encoding process to achieve decoding.

Spherical panoramic images are generally represented by a three-dimensional sphere, and the coordinates on it are mostly following the rules of right-hand operation and are represented by a three-dimensional coordinate system. The points on the sphere can refer to the marking method of the three-dimensional scanner and perform punctuation on several latitudes and longitudes [11]. Longitude takes the $x$ -

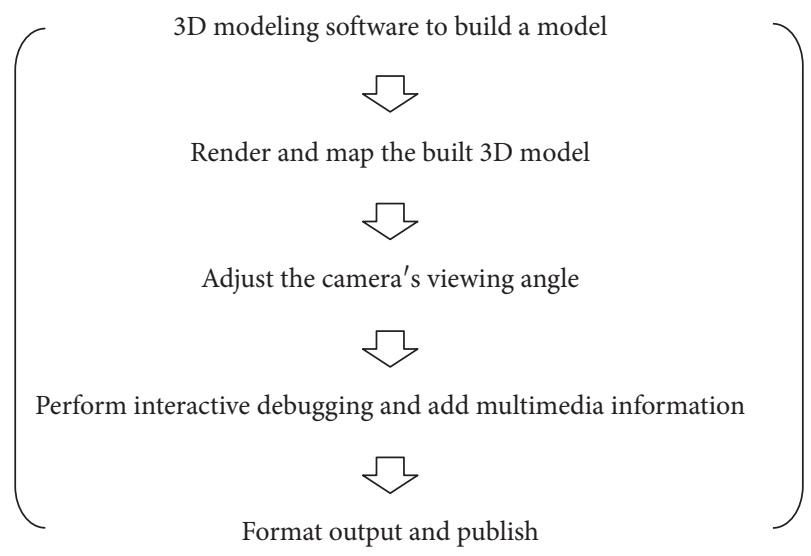

FIGURE 3: Diagram of the process of VR technology for display design.

axis as the coordinate direction and uses counterclockwise rotation, and the rotation angle value is positive. On the contrary, when the rotation angle is clockwise, the rotation angle is negative. The longitude value is represented by $\pi$, and the value range is $[-\pi, \pi]$. Taking the equator on the sphere as the coordinate, the latitude uses the $Y$-axis as the coordinate direction, the coordinate point moves toward the north pole, and the angle value is positive. On the contrary, if the coordinate point moves to the south pole, the angle value is negative. Therefore, the latitude value range is $[-\pi / 2, \pi / 2]$. The coordinates of a point on the unit sphere can be expressed by latitude and longitude coordinates $[\Phi, \theta]$ or by three-dimensional coordinates $(X, Y, Y)$, which are expressed as follows:

$$
\begin{aligned}
& X \cos \varphi \cos \theta, \\
& Y=\sin \varphi, \\
& Z-\cos \varphi \sin \theta .
\end{aligned}
$$

Through the above analysis, the essence of panoramic image projection is to project the panoramic image frame and all pixels on the spherical texture in a certain way and then convert the $3 \mathrm{D}$ video image into a $2 \mathrm{D}$ plane video frame image. That is to say, to realize the VR technology to display the artifacts in the virtual space, it is necessary to establish a geometric model, map the coordinate points on the spherical surface to the surface of the geometric body, complete the spherical video projection to the two-dimensional plane [12], and transform and rotate the texture pixels on each surface of the geometry.

From another level, in the panoramic video image, the spherical panoramic image can also be realized according to the idea of equidistant cylindrical projection. The method is to use coordinate points of the same value to expand the latitude line data on the sphere and map it on a two-dimensional plane to obtain a rectangular video. In the unified plane coordinate system, the plane coordinate points use $U$ and $V$ to represent the values, and the range of values is $(0,1)$. The latitude and longitude coordinate points on the spherical surface are $(\theta, \Phi)$; thus, the spherical panoramic image to the plane image conversion, the corresponding 
method of its value, is obtained by a formula using the coordinates of the plane point $(U, V)$ (formula (2)). Then, the three-dimensional point coordinates $(X, Y, Y)$ on the spherical surface are calculated by formula (1).

$$
\begin{aligned}
& \theta=2 \pi \times(U-0.5), \\
& \theta=\pi \times(0.5-V) .
\end{aligned}
$$

If any point $(X, Y, Y)$ in the three-dimensional space is inversely converted into a two-dimensional plane point, the longitude and latitude values $(\Phi, \theta)$ can be obtained by formula (1), and the value of the plane point $(U, V)$ can be obtained by formula (2) [1].

3.5. Display Mode of the Ceramic Space Scene. VR technology is involved in the design of ceramic virtual exhibition space; one not only can feel the atmosphere of the scene but also can better observe the objects. This method is more suitable for ceramic products and artworks, allowing viewers to fully and profoundly reflect the performance of ceramic products in the space atmosphere. It can not only display ceramic products and artworks but also use other ceramic categories, such as architectural ceramics, industrial ceramics, and special ceramics. However, from the perspective of display purposes, ceramic products and artworks require a panoramic view, object body, and scene. Several display methods cooperate to realize viewing in virtual space. First of all, in the environment of panoramic display mode, the displayed objects are relatively fixed and can only be moved 360 degrees through the lens. During the movement of the lens, the viewer will appreciate the complete display space scene as the lens moves. Secondly, look around the 360-degree landscape from a height. Secondly, use the object display. In this display mode, the lens is relatively fixed. By rotating the object up, down, left, and right, the viewer can observe the object; the last is the scene display, which is both the object and the lens. Generate movement, such as setting multiple observation points; you can walk from one observation point to another; that is, you can watch the scene as well as the object, and you can feel the object from the atmosphere of the scene.

Regardless of the way of display, the show requires multiple observation points; through VR technology and interactive design in the virtual software with corresponding background music, the viewer can comfortably roam from one display scene in the virtual space to another display scene; in the virtual display space, the viewer can not only appreciate the whole object but also magnify the part of the object and appreciate the local details of the object; under the background music rendering, the viewer is completely immersed in the appreciation in the charming space of ceramics. At the same time, the viewer can zoom in and out, move up and down, watch from multiple angles, and change the glaze color or decorative style of the ceramics in the virtual design [1]. Especially in the postepidemic era, some public exhibition spaces such as museums have been affected by the epidemic, and the flow of people has been correspondingly restricted. The introduction of VR technology into the design and display of ceramic products effectively solves the limitation of not being able to go to the museum to watch the real scene but also narrates the distance between people and the objects and increases the enthusiasm of the public to participate in ceramic design.

\section{Advantages of VR Technology for Chinese Ceramics Exhibition}

4.1. VR Technology Used to Display Ceramic Products Helps Spread China's Ceramic Culture. China has a long ceramic culture and there are so many ceramic-producing areas in China. The audience cannot visit all the ceramic-producing areas for on-the-spot inspections to experience the characteristics of the utensils in different producing areas. In addition, the porcelain is fragile, which is displayed in the real space. The above brought certain difficulties, which made it difficult for Chinese ceramics to be accepted by the masses, resulting in the inadequate dissemination of ceramic culture. The emergence of VR technology provides a new way for the dissemination of ceramic culture, breaks through the limitations of traditional communication methods, and adds a boost to the dissemination of ceramic culture. Relevant units and social organizations can use VR technology to spread ceramic culture, make full use of the characteristics of VR technology, and display ceramic culture to the masses in a novel way so that ceramic culture can be better spread. The masses can also make full use of VR technology, VR cultural promotion center, or their VR equipment to understand the ceramic culture.

\subsection{The Viewer Has a Diversified Sensory Experience for} Ceramic Display under the VR Field of Vision. The traditional display method mainly adopts real-time display, which consumes a lot of manpower and material resources in the preparation process, and it is also easy to cause damage to the ceramics. In addition, the flow of people is often restricted during the exhibition, so that the viewer cannot get the sensory experience of the appointment. In the current era of information development, traditional display methods cannot meet the needs of the masses and therefore cannot meet the needs of ceramic display and ceramic culture dissemination. VR technology has unique advantages and strong interactivity, which can effectively strengthen the interaction between the experiencer and ceramic culture. The VR scene designer can construct virtual historical characters in the VR scene, interact with the experiencer, and enhance the experience of the experiencer. At the same time, VR technology can present sensory sensations such as hearing and touch, enabling the experiencer to obtain a diversified sensory experience.

\subsection{VR Technology Makes the Audience Situation of Ceramics} Exhibition Tend to Be Good. In recent years, with the strong support of the government and relevant departments, intangible cultural bases and ceramic industry inheritance centers have been established everywhere, and ceramics can be displayed and disseminated to the maximum extent. As 


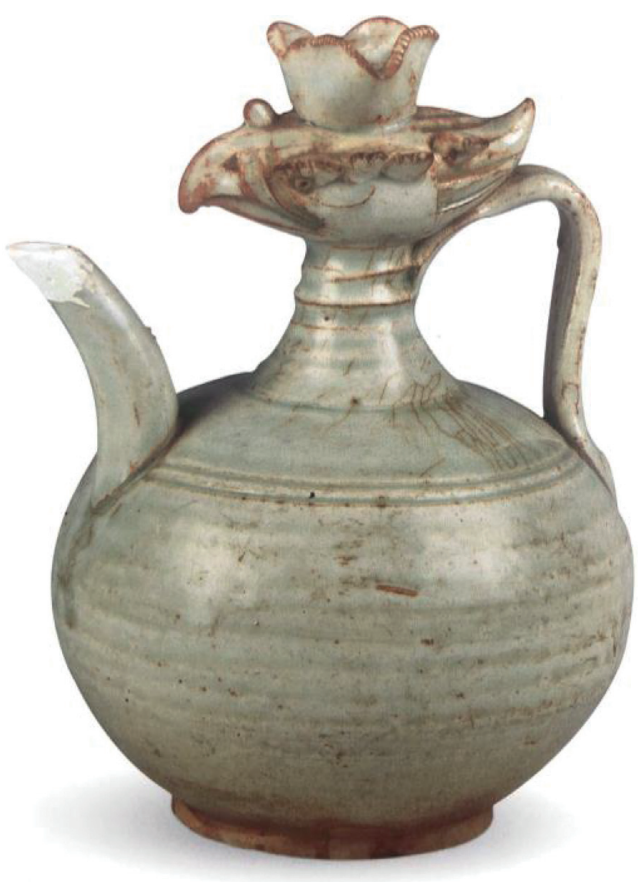

Figure 4: The bluish-white glazed phoenix head pot of Fanchang kiln.

The schematic diagram of the public's awareness of Chinese ceramics and VR technilogy

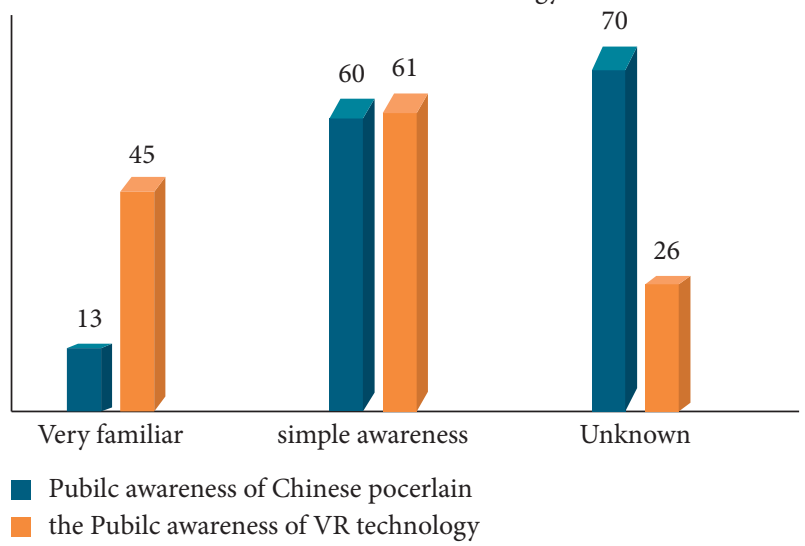

FIgURE 5: The cognitive comparison of the number of people.

the broad masses of the people, we have the responsibility and obligation to promote, protect, and inherit Chinese traditional culture. Compared with the encouraging, compulsory, and guiding propaganda methods adopted by the government and relevant departments, mass groups have the advantages of wide dissemination and high information openness. This result has made the public actively appreciate the sensory charm brought by ceramic culture and spontaneously join in the promotion of Chinese ceramic culture, becoming the main force of ceramic culture promotion. In the process of studying the bluish-white porcelain of Fanchang kiln, the author took the bluish-white glazed phoenix head pot (Figure 4) as the display object, explained the characteristics of the phoenix head pot in detail to the audience in the virtual scene of VR, and analyzed the main points of the design of the utensil. After obtaining this information, he quickly became a disseminator, making Fanchang kiln blue and white glaze a star display product. Based on this, to obtain audience data, the author publishes questionnaire information through his social circle, museum visitors, and student groups. As shown in Figure 5, among the 100 users, 40 are women, $40 \%$, and 60 are men, accounting for $60 \%$. Most of them are 21-40 years old, followed by 41-60 years old, under 20 years old, and 61 years old and above. The results show that the use of VR technology in the display of ceramic products has increased the number of audiences to a certain extent, and there is a tendency to increase.

\section{Conclusion}

With the development of science and technology, VR technology as a display medium provides an opportunity for the development of the ceramic product industry. The use of VR technology for display allows people to perceive products as if they have entered a real scene, and they can also interact with products in the scene at any time. This method not only plays a role in protecting ceramic products but also provides a superior path for the spread of ceramic culture. VR technology has realized the essential characteristics of different ceramic products and the physical functions of ceramic products. Take targeted solutions to explore the most suitable virtual display performance methods for different types of ceramic products, and accurately express the display content and display of different types of ceramic products. The key point is to realize the viewing and interaction of ceramic products from different angles, and the virtual display method can be applied to the production of the virtual display through the network and interactive projection. Let VR technology make up for the disadvantages of traditional exhibition halls. In terms of interactivity, both customers and merchants can obtain accurate information and promote the development of the ceramic industry.

\section{Data Availability}

Data sharing is not applicable to this article as no datasets were generated or analyzed during the current study.

\section{Conflicts of Interest}

The authors declare that they have no conflicts of interest.

\section{Acknowledgments}

This article was funded by the Anhui Province Outstanding Top Talent Project (gxbjZD2021005).

\section{References}

[1] X. Hu and L. Zhang, "VR technology in bluishwhite porcelain display design of Fanchang kiln," IEEE Access, Article ID 3019466, 2020.

[2] Y. Wang, Z. Zhao, and W. Song, "Virtual ceramic product design based on VR technology," Packaging Engineering, vol. 00, no. 12, pp. 209-211, 2007. 
[3] Z. H. Wang, "Application of VR technology in modern ceramic display design," Youth Era, vol. 46, no. 22, pp. 47-48, 2018.

[4] Xi Tao, "On virtual reality technology (VR) leading the development of industrial design," Packaging Engineering, vol. 28, no. 7, pp. 124-126, 2007.

[5] M. C. Ward, “The 'new listening': richard wagner, nineteenthcentury opera culture, and cinema theatres," Nineteenth Century Theatre and Film, vol. 43, no. 1, pp. 88-106, 2016.

[6] Y. Wang and X. Hu, "Wuju opera cultural creative products and research on visual image under VR technology," IEEE Access, vol. 8, pp. 161862-161871, 2020.

[7] W. Yanqun and L. Yanghua, "Design and practice of cultural creative product of Training Meilin opera," Package Engineering, vol. 38, no. 22, pp. 203-206, 2017.

[8] A. Karim, "Multi-layer masking of character data with a visual image key," International Journal of Computer Network and Information Security, vol. 9, no. 10, pp. 41-49, 2017.

[9] W. Liu, X. Meng, and J. Zhang, "Application research of panoramic roaming based on VR technology in ice and snow sculpture display design," IPPTA: QuarterlyJournal of Indian Pulp and Paper Technical Association, vol. 30, no. 6, pp. 360-363, 2018.

[10] M. Wang, X.-Q. Lyu, Y.-J. Li, and F.-L. Zhang, "VR content creation and exploration with deep learning: a survey," Computational Visual Media, vol. 6, no. 1, pp. 3-28, 2020.

[11] G. Xiao, M. Wu, Q. Shi, Z. Zhou, and X. Chen, "DeepVR: deep reinforcement learning for predictive panoramic video streaming," IEEE Transactions on Cognitive Communications and Networking, vol. 5, no. 4, pp. 1167-1177, 2019.

[12] W.-T. Lee, H.-I. Chen, M.-S. Chen, I.-C. Shen, and B.-Y. Chen, "High-resolution 360 video foveated stitching for real-time VR," Computer Graphics Forum, vol. 36, no. 7, pp. 115-123, 2017. 